\title{
Numerical Analysis of the Influence of Foundation Pit Blasting on a Nearby Metro Tunnel
}

\author{
Haibo Wang (D, Yaoyao Wang, Mengxiang Wang, and Qi Zong \\ School of Civil Engineering and Architecture, Anhui University of Science and Technology, Huainan 232001, Anhui, China \\ Correspondence should be addressed to Haibo Wang; wanghb_aust@163.com
}

Received 15 June 2021; Revised 11 August 2021; Accepted 12 August 2021; Published 7 September 2021

Academic Editor: Qi Zhao

Copyright (C) 2021 Haibo Wang et al. This is an open access article distributed under the Creative Commons Attribution License, which permits unrestricted use, distribution, and reproduction in any medium, provided the original work is properly cited.

\begin{abstract}
It is important to guarantee the safety of adjacent underground structures during the foundation pit blasting excavation of modern city construction. The blasting excavation construction of a large, deep foundation pit near an existing metro station of Guangzhou Metro Line 3 is used as the example in this study. Based on blasting vibration field test results, the influence of blasting dynamic load on the lining of an adjacent metro tunnel is numerically analyzed in simulation using Fast Lagrangian Analysis of Continua 3D (FLAC3D), and the relationships between the blasting vibration velocity and stress and the displacement of the metro tunnel lining are obtained. The results show that the stress of lining structure is within the allowable range under the experimental blasting conditions, the lining displacement increases linearly with the applied dynamic vibration velocity, and the vertical displacement of the lining is more obviously affected than the horizontal displacement by the dynamic load. This study can be used as a basis for the control of blasting vibration in a complex urban environment. Its practical application shows that the proposed blasting plan and parameters are reasonable and effective.
\end{abstract}

\section{Introduction}

Blasting is an efficient and fast excavation method for hard rock mass, and it has been widely used in the excavation of urban building foundation pits. With the continuous construction of urban metro in China, dangers associated with the blasting excavation of foundation pits adjacent to existing urban metro often arise. Most metro tunnels are lined with reinforced concrete segments. When the blasting vibration exceeds a certain limit, it can cause catastrophic damage to the lining. In order to ensure the safety and stability of the adjacent metro tunnel, the influence of the blasting vibration should be evaluated, and potentially damaging effects should be minimized. The abovementioned issue is a key technical issue in realizing the safe and efficient implementation of build foundation pit construction in complex urban environments.

At present, research on the response characteristics of foundation pit on a metro tunnel has been widely considered by scholars [1-5]. However, most of these studies focus on the displacement and stability of foundation pit, and less attention has been paid to blasting engineering and blasting seismic wave influence on a metro tunnel. Xia et al. researched the impact of the cavity effect on tunnel excavation blasting vibration by a series of blast tests. The results suggested that the cavity effect on the ground only worked within a limited scope in shallow tunnel excavations, and an empirical equation was proposed to calculate the amplifying coefficient [6]. Koneshwaran et al. used a fully coupled technique involving smooth particle hydrodynamics and finite element techniques to investigate the blast response of segmented bored tunnels [7]. El Koursi et al. devised technological improvements to the structure and critical systems of the vehicle and tested in real situation, focused particularly on the behavior of people in blast situation, and presented the related improvements of the vehicles' design in order to improve resilience and survivability to blast [8]. Shin et al. modified the detonation pressure equation based on the results of field tests. Tunnel behavior due to blastinduced vibration was investigated in terms of particle velocity, displacement, and stress of the linings, and guidelines for a blast protection zone were proposed [9]. Ozer analyzed 
the field-testing results of ground vibration induced by blasting during the construction of the Istanbul KadikoyKartal metro tunnel. The empirical relationships between the scaled distance and peak particle velocity were established in order to predict the influence on neighboring buildings and structures [10]. Ocak and Bilgin studied the performance of a roadheader, impact hammer and drilling and blasting method in the excavation of metro station tunnels in Istanbul and gained machine utilization time and average net cutting rate of each machine [11]. De et al. studied the effects of a surface explosion through a combination of physical model tests and numerical modeling. The influence of the barrier thickness in reducing the explosion-induced strains, stresses, and pressures on the tunnel was analyzed [12]. Park and Jeon proposed an air-deck method to reduce blast-induced vibration in the direction of tunneling, and its effect was investigated through numerical and experimental studies [13]. Masoudi and Sharifzadeh studied the reinforcement selection for deep, high-stress tunnels in preliminary design stages using a ground demand and support capacity approach. This covered methods to measure the energy dissipation capacities of rockbolts, and the applicable range of each type of rockbolt was presented [14]. Feldgun et al. presented a comprehensive approach to simulate the effects of an explosion in a buried infrastructure tunnel on the soil surface and nearby tunnels. This simulation considered all stages of this complex process, including the detonation of the internal explosive charge, the shock wave propagation through the air in the tunnel, and the following interaction with the cavity lining. The simulation then examined the soil-structure dynamic interaction [15]. Lin et al. proposed a novel fuzzy model for identifying high-risk factors during excavations in urban karst geological environments [16]. Qin and Zhang analyzed the influence of the maximum charge on the vibration speed of the existing tunnel by comparing the influence of adjacent tunnel blasting team on the existing tunnel simulated by ANSYS LS-DYNA with the actual monitoring results [17]. Jiang et al. focused on safety of buried pressurized gas pipelines subject to blasting vibrations induced by metro foundation pit excavation $[18,19]$. In order to control boulder deep-hole blasting-induced vibrations to a neighboring metro shaft, the damping hole and the isolation hole and changing the charging layout of the blasting areas were applied [20]. Also, Liu et al. optimized blasting scheme of metro stations subsurface excavating oblique through pile foundations of existing elevated light rail lines [21].

This paper focuses on the blasting excavation of the Taiguhui foundation pit in Guangzhou, China. This pit is adjacent to Shipaiqiao Station of Guangzhou Metro Line 3. Vibration of blasting excavation with small quantity of explosives is tested, and a typical vibration curve of blasting seismic wave is gained. The three-dimensional numerical calculation model of rock mass and metro lining is established by the finite difference software FLAC3D. On that base, the dynamic response characteristics of metro lining and rock mass under different load conditions are calculated and analyzed. The relationships between maximum displacement and vibration velocity of lining under different dynamic load conditions are calculated and analyzed. The prediction formula of metro lining PPV is established, which can guide the parameters' design of foundation pit blasting.

\section{Overview of Foundation Pit Blasting Construction}

2.1. General Engineering Situation. The foundation pit is at the northwest corner of the intersection of Tianhe Road and Tianhe East Road in Guangzhou City, China. The project consists of a podium building and three towers of 30, 45, and 31 stories. The building basement is four stories deep, and the foundation pit is $260 \mathrm{~m} \times 160 \mathrm{~m}$. The excavation depth of the foundation pit is $27 \mathrm{~m}$. The enclosure structure of the foundation pit is a continuous concrete wall. The building's foundation is the enlarged foundation of a manual digging pile, the diameter of the building pile foundation ranges from 1.5 to $2.8 \mathrm{~m}$, and the pile diameter of the towers is larger than that of the podium building. According to the geological data of this project, it would be difficult to excavate the foundation pit and pile foundation of the buildings in the middle weathered rock stratum using a machine, so a blasting method is needed.

The foundation pit is in a complex environment. The east side is on Tianhe East Road, which is a busy road. The south side is on Tianhe Road, which is a main road with many municipal pipelines laid underneath. Shipaiqiao Station of Guangzhou Metro Line 3 is about $32-36 \mathrm{~m}$ from the side of the foundation pit. It is about $10 \mathrm{~m}$ between the pedestrian channel and the pit. Therefore, the harmful effect of the blasting construction must be controlled.

The surrounding environment is shown in Figure 1. The enclosure structure and the rock and soil conditions of the foundation pit are shown in Figure 2.

The geological survey results show that the buried depth of static groundwater level is $0.8 \sim 4.6 \mathrm{~m}$. The main aquifers of the site are the pore water in miscellaneous fill soil, silt layer, and bedrock fissure water. The pore water in miscellaneous fill is perched water, and the water level fluctuates with seasons. The silt layer is confined water, which is vertically infiltrated by the upper stagnant water and laterally supplied by this layer. Bedrock fissure water mainly exists in strongly weathered and moderately weathered rock strata, which belongs to confined water and is mainly supplied by fissure water laterally. Meanwhile, lateral seepage is the main form of drainage.

2.2. Blasting Program. The excavation blasting construction mainly includes manual excavation pile blasting and foundation pit blasting of stone. Different blasting methods are adopted according to their respective engineering characteristics.

2.2.1. Manual Digging Pile Blasting Plan. Short-hole bench blasting can be used for the weathered bedrock section of a manual hole digging pile. The holes should be arranged as central cut, caving, and contour holes. Accounting for the blasting damage and convenience of construction, the 


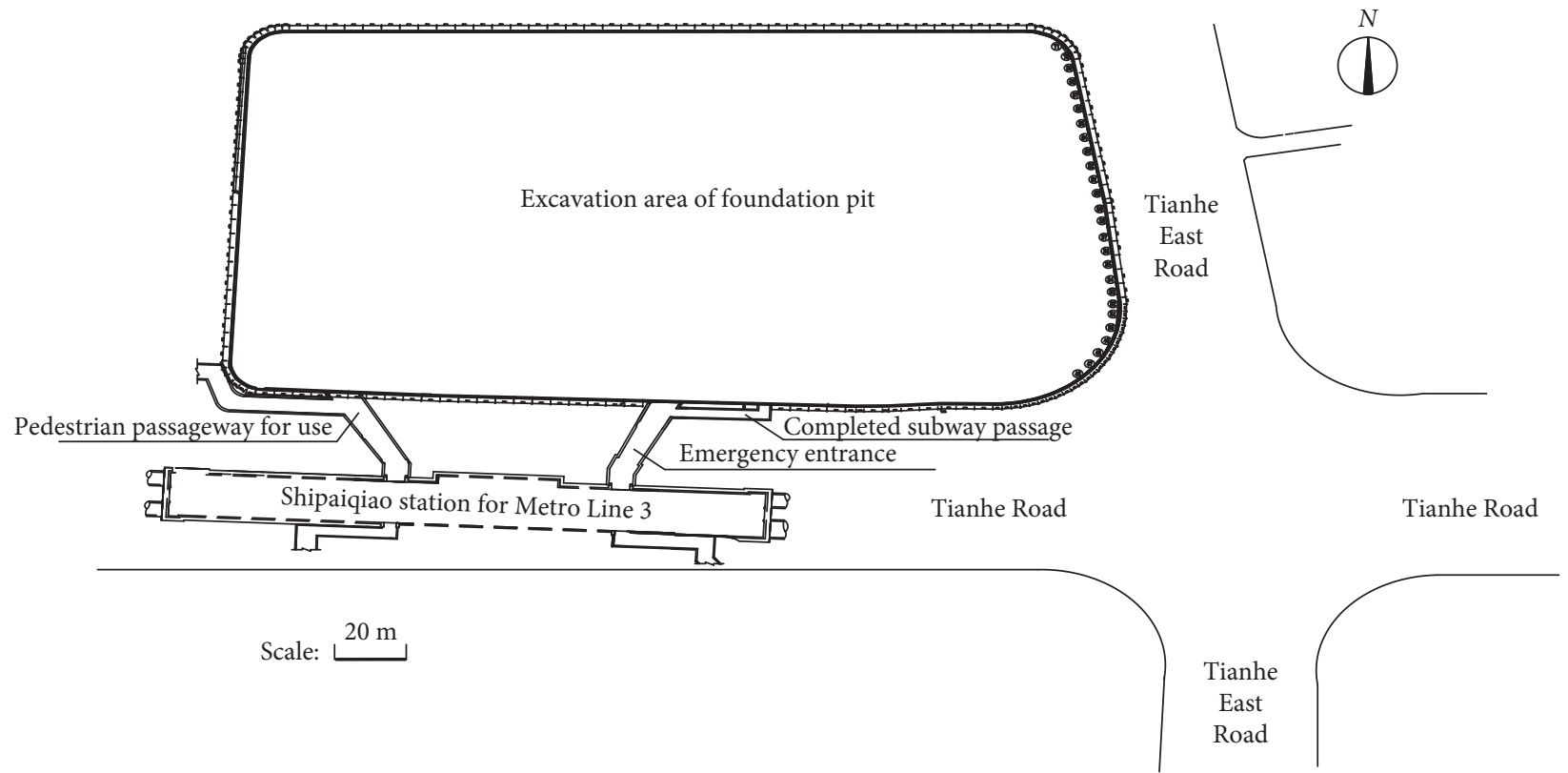

FIGURE 1: Surrounding engineering environment.

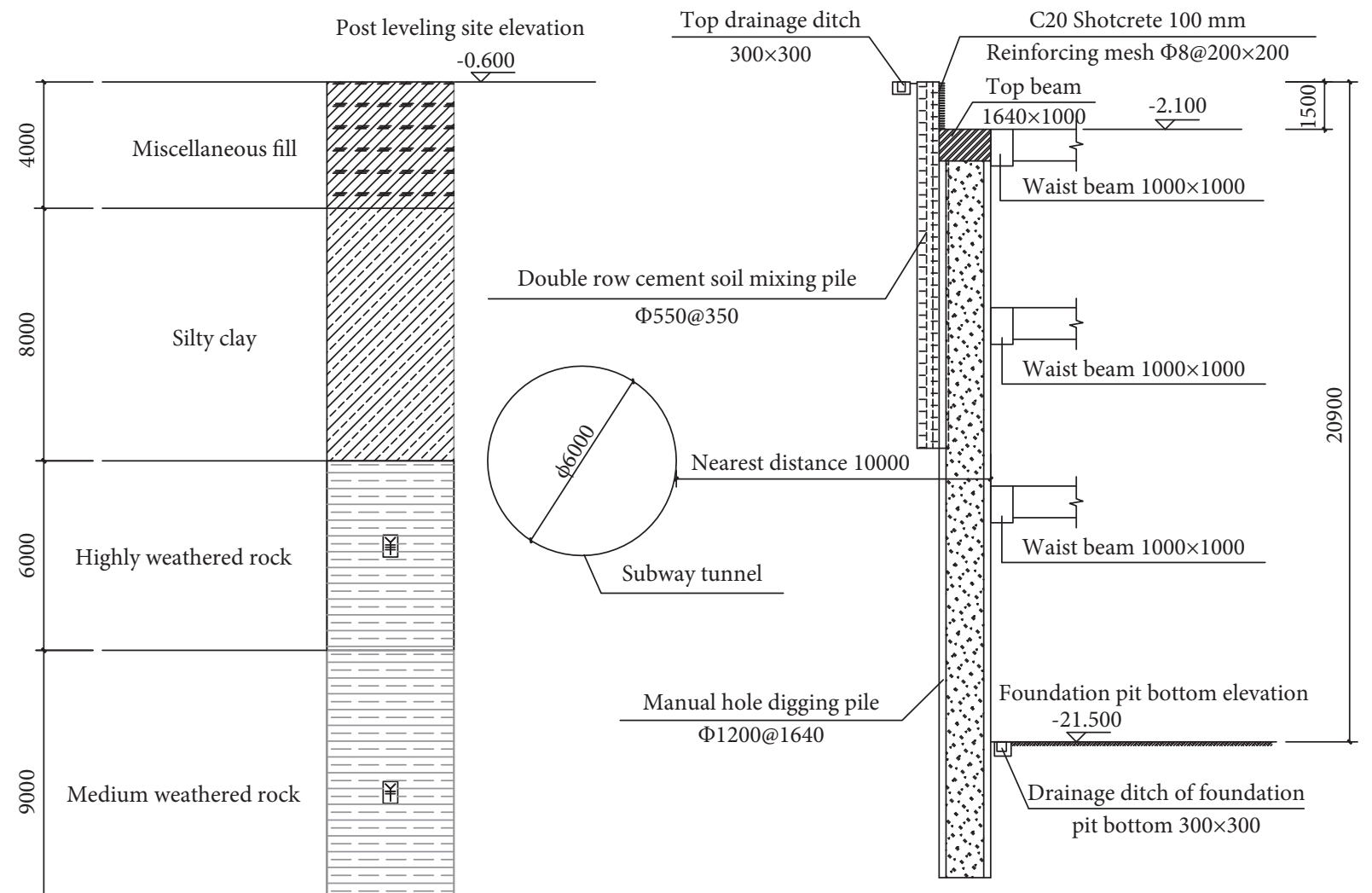

Note: In this picture, unit of elevation is metre (m), unit of other

Slightly weathered rock

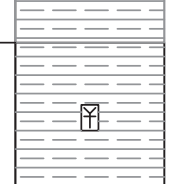
size is millimeter $(\mathrm{mm})$

Figure 2: Enclosure structure and rock and soil conditions of the foundation pit. 
opening position of the contour hole is $0.2-0.3 \mathrm{~m}$ from the design outline. A millisecond-delay detonator is used to achieve millisecond-controlled blasting, and protective measures should be taken to cover the pile wellhead to avoid flying stones.

2.2.2. Foundation Pit Blasting Plan. The foundation pit is in the downtown area. There are buildings, roads, and underground pipelines around the blasting site. Blasting vibration and flying stones should be strictly controlled, and short-hole bench blasting with $2.0-3.0 \mathrm{~m}$ in depth should be adopted.

According to the rock strata exposure condition of the foundation pit, the blasting area is divided into two regions. The position of the bench in the center of the foundation pit, the drill, and the blast with a hand-held drilling rig are selected to form a small bench, around which blasting excavation occurs. Because the resistance lines of the bench blasting all point to the wider area of the foundation pit center, it is beneficial to reduce the production of flying stones.

To ensure the safety of nearby metro station, harmful effects of blasting must be controlled, a protective layer should be reserved at a distance of $2.0 \mathrm{~m}$ from the retaining structure of the foundation pit, and the rock should be broken by a crushing hammer to ensure the stability of the retaining structure.

The excavation sequence of the foundation pit is shown in Figure 3. For blasting vibration controlling, A region and $\mathrm{B}$ region were divided into several detonations.

2.3. Blasting Parameters. Air leg drill with the model of YT28 is adopted for blast hole drill, and the diameter of the drill hole is $42 \mathrm{~mm}$.

Dynamite: emulsion explosive is used, and the diameter of the roll is $32 \mathrm{~mm}$.

The bench height of short-hole bench blasting is $2-3 \mathrm{~m}$, the borehole depth is $2.2-3.2 \mathrm{~m}$, the borehole spacing is $1.0-1.2 \mathrm{~m}$, the row spacing is $0.9-1.2 \mathrm{~m}$, and the front and rear rows are formed using a plum blossom pattern hole.

The blasting hole depth of the manual digging pile is $1.0 \mathrm{~m}$, the cutting hole and hollow hole depths are both $1.2 \mathrm{~m}$, and the blasting hole circle distance ranges from 0.3 to $0.5 \mathrm{~m}$.

A millisecond detonator and a hole bottom continuous charge structure are used, a detonation charge pack is placed in the middle or lower part of the charge section, and the noncharge section is plugged with rock powder.

2.4. Blasting Vibration Monitoring and Research. In the construction site, the upper ground is soil and the lower ground is weathered rock, causing the propagation of the blasting vibration wave to attenuate rapidly. The blasting vibration is monitored by the vibrations of NUBOX-6016 according to a field test and regression analysis, and the blasting vibration attenuation law is

$$
v=160\left(\frac{\sqrt[3]{Q}}{R}\right)^{1.7}
$$

where $v$ is the vibration velocity, $Q$ is the charge quantity, and $R$ is the distance between the blasting area and blasting vibration measuring point.

A typical curve of the vibration velocity time history is shown in Figure 4.

For the blasting construction of the surrounding buildings and roads, the allowable vibration velocity of Guangzhou City is less than $2.0 \mathrm{~mm} / \mathrm{s}$.

The maximum amounts of charge at different distances, as calculated by equation (1), are shown in Table 1 .

The minimum distance between the exit passage entrance of Shipaiqiao Station of Metro Line 3 and the side of the foundation pit is approximately $10 \mathrm{~m}$. According to Table 1, the maximum charge per blasting section is $0.44 \mathrm{~kg}$, which seriously affects the construction schedule. However, the allowable velocity of the traffic tunnel in the technical specification for retaining and protection of building foundation excavations in Guangzhou area is less than $15.0 \mathrm{~cm} / \mathrm{s}$ [22]. According to equation (1), the maximum amount of the corresponding single section is $15.34 \mathrm{~kg}$, which is quite different from the values in Table 1 .

Based on this, the stress and deformation of the metro tunnel under different blasting dynamic loads are analyzed with FLAC3D software according to the typical time history curve of the vibration velocity obtained from the test. The numerical simulation results can guide blasting engineering practice.

\section{Numerical Simulation Analysis of the Influence of a Blasting Dynamic Load on an Adjacent Tunnel}

\subsection{Basic Assumptions.}

(1) The tunnel lining is a whole reinforced concrete structure; the formation distribution of the rock and soil is stratified

(2) The tunnel lining is in close contact with the surrounding strata

(3) Ignoring the influence of the rheological characteristics of the rock and the soil mass, as well as joints, fractures, and possible water in the rock mass, the rock mass and lining are taken as a uniform, continuous, isotropic medium

\subsection{Calculation Model and Parameters}

3.2.1. Calculation Model. The metro tunnel outside diameter is $6.0 \mathrm{~m}$, the inner diameter is $5.4 \mathrm{~m}$, the lining thickness is $0.3 \mathrm{~m}$, the tunnel center buried depth is $12 \mathrm{~m}$, and the space between the tunnel center and the foundation pit edge is calculated as $10 \mathrm{~m}$ for safety. The relationships are shown in Figure 5 . 


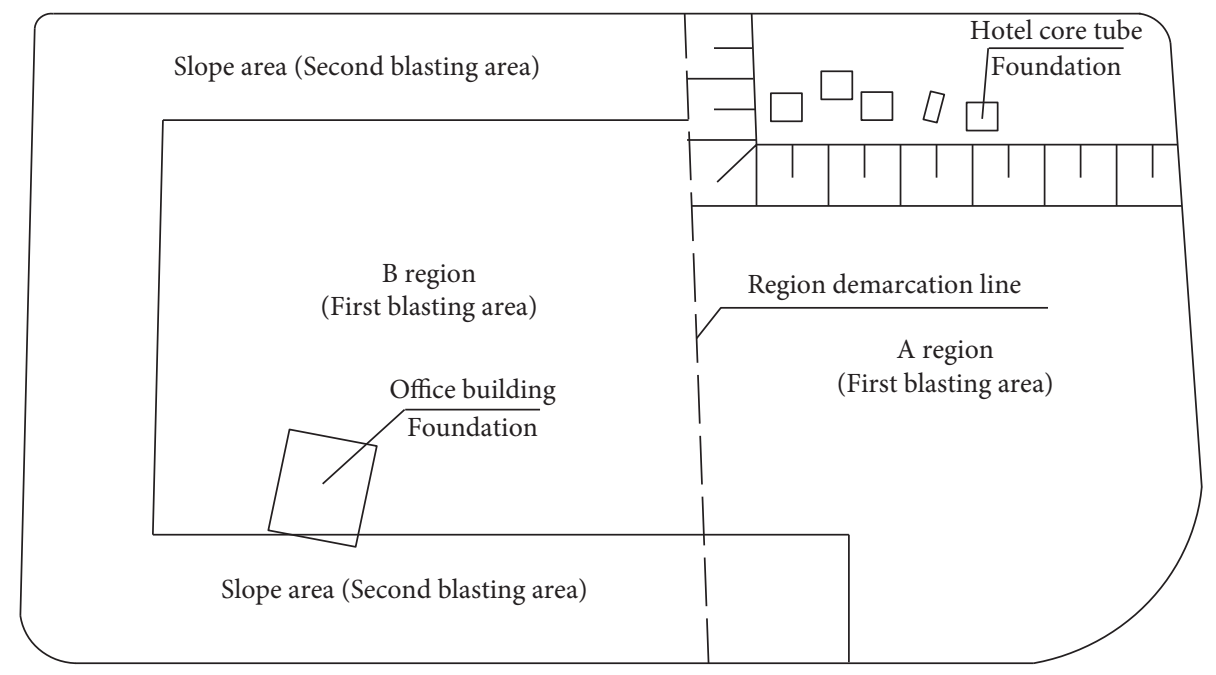

Figure 3: Blasting subsequence of the foundation pit.

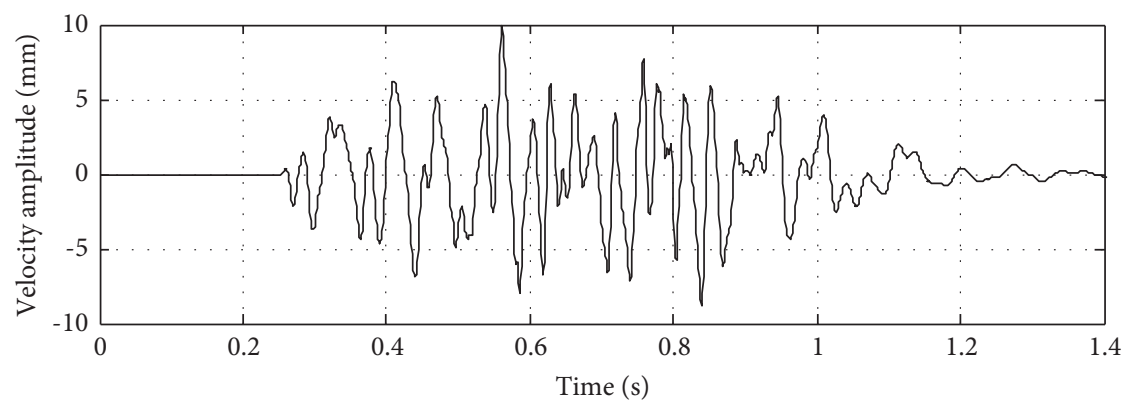

FIgUre 4: Typical vibration curve of a blasting seismic wave.

Table 1: Permitted maximum charge of one section.

\begin{tabular}{lccccccc}
\hline Distance $(\mathrm{m})$ & 10 & 12 & 16 & 18 & 20 & 25 & 30 \\
\hline Charge $(\mathrm{kg})$ & 0.44 & 0.76 & 1.79 & 2.56 & 3.51 & 6.85 & 11.83 \\
\hline
\end{tabular}

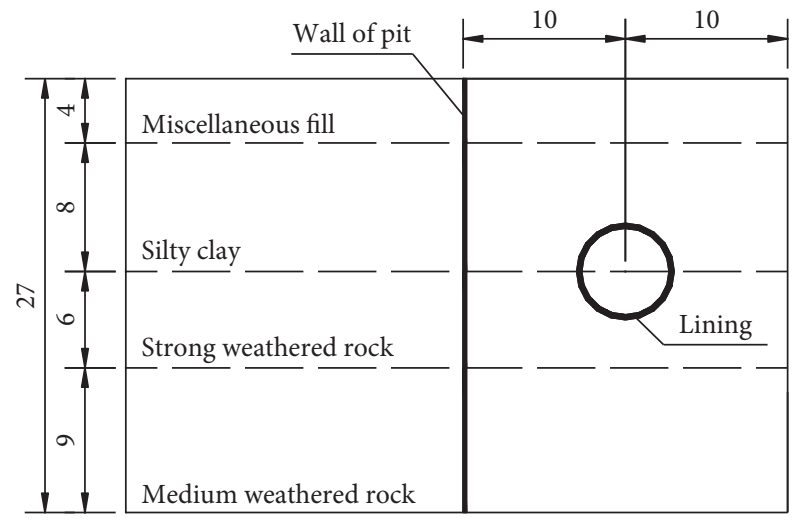

FIgURE 5: Position relationships between the tunnel and the pit (unit: $\mathrm{m}$ ).

The effect of the blasting dynamic load on the metro tunnel lining, regardless of the foundation pit supporting structure, is modeled using only the tunnel lining, soil, and rock mass, as shown in Figure 6.
The coordinate system in the calculation model is set according to the following conditions. The center of the metro tunnel is taken as the original point $(0,0,0)$. The trending direction of the tunnel is set as the $Y$ axis, and the direction oriented perpendicular to the paper is set as positive. The direction perpendicular to the tunnel trending direction is set as the $X$ axis, and the right direction is set as positive. The vertical direction of gravity is set as the $Z$ axis, and the upward direction is set as positive. The model size is $10 \mathrm{~m} \times 20 \mathrm{~m} \times 27 \mathrm{~m}$ (trending length $\times$ width $\times$ height). According to the abovementioned parameters, the calculation model of the metro tunnel and rock mass is established as shown in Figure 6.

During the calculation, the rock mass is modeled using the Mohr-Coulomb elastic-plastic constitutive model. The principal stress in three directions of the rock and soil can be assumed to be $\sigma_{1}, \sigma_{2}$, and $\sigma_{3}$, with $\sigma_{1}>\sigma_{2}>\sigma_{3}$, so the Mohr-Coulomb yield criterion is expressed as

$$
\left\{\begin{array}{l}
f^{s}=\sigma_{1}-\sigma_{3} N_{\phi}+2 c \sqrt{N_{\phi}} \\
f^{t}=\sigma_{3}-\sigma^{t}
\end{array}\right.
$$

where $\sigma_{1}$ is the maximum principal stress, $\sigma_{3}$ is the minimum principal stress, $\varphi$ is the internal friction angle, $c$ is the cohesion, $\sigma^{t}$ is the tensile strength, $\sigma_{\max }^{t}=c / \tan \varphi$, and $N_{\phi}=1+\sin (\phi) / 1-\sin (\phi)$. When $f^{s}=0$, the material will 


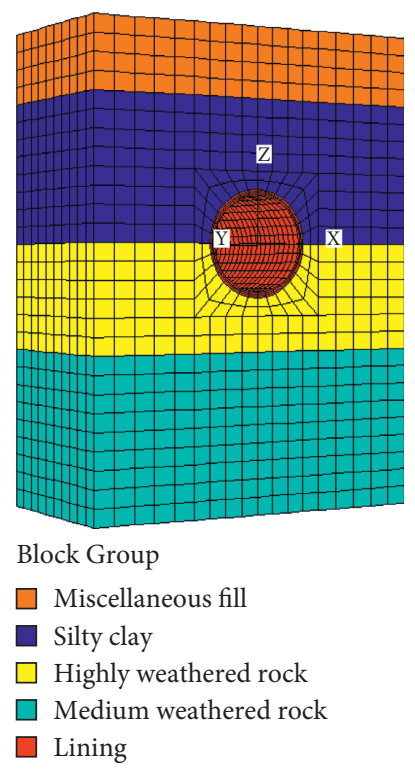

Figure 6: Calculation model of FLAC3D.

experience shear failure; when $f^{t}=0$, the material will experience tensile failure.

3.2.2. Boundary Condition. The FLAC3D dynamic calculation is based on the results of a static calculation whose calculation boundaries are set as follows. The $Z$-direction constraints are imposed on the bottom of the model, and the other five surfaces are set as free boundaries. During the dynamic calculation, the $X$-direction constraints are imposed on either side of the model for incident wave absorption. The $Y$-direction constraints are imposed in the tunnel trending direction. The Z-direction constraints are imposed for the model. The stress boundary condition is controlled using the upper section of the model, and the static boundary model is used for dynamic reflection boundary conditions.

3.2.3. Selection of Damping. When excavation blasting is performed, the blasting seismic wave propagation in the formation causes the vibration energy to gradually decay due to damping, and the rock particle vibration eventually stops. During the dynamic analysis, Rayleigh damping is used, with a minimum critical damping ratio of 0.1 and a minimum center frequency of $10 \mathrm{~Hz}$.

3.2.4. Application of Blasting Seismic Wave Load. A separation of the wave and field technique is adopted for the applied dynamic load. The blasting seismic load is translated to an equivalent load and applied directly to the artificial boundary. A blasting vibration velocity wave can be transformed to a stress wave as follows [11]:

$$
\begin{gathered}
\sigma_{n}=2\left(\rho C_{p}\right) v_{n}, \\
\sigma_{s}=2\left(\rho C_{s}\right) v_{s},
\end{gathered}
$$

where $\sigma_{n}$ is the applied normal stress, $\sigma_{s}$ is the applied shear stress, $\sigma$ is the density of the transmission medium, $C_{p}$ is the longitudinal wave propagation of the medium, $C_{s}$ is the transverse wave velocity, and $v_{n}$ and $v_{s}$ are, respectively, the normal and tangential particle vibration velocities of the imposed blasting vibration load.

In Figure 4, the peak velocity of the curve is about $1.0 \mathrm{~cm} /$ $\mathrm{s}$, and four kinds of construction conditions are designed. The blasting vibration velocity amplitude of each condition and the corresponding maximum charge quantity can be calculated according to equation (1). The calculated results are shown in Table 2.

Because of the blasting construction on the medium weathered stratum in Figure 1, the dynamic load is applied in the lower-left (medium weathered rock) section of the model. According to equations (3) and (4), the blasting vibration velocity changes to a stress wave, the blasting seismic wave curve of Figure 4 is set as a benchmark, and the dynamic loads of different working conditions are multiplied by the appropriate factors.

3.2.5. Physical and Mechanical Parameters. The physical and mechanical parameters of the lining, soil, and rock mass for the numerical calculation are shown in Table 3.

\section{Numerical Simulation Results and Analysis}

4.1. Numerical Simulation Results and Analysis of the Static Load. According to the abovementioned model and parameters, the displacement and stress contour charts of the stratum and metro tunnel lining in static load are determined, as shown in Figures 7 and 8.

Figure 7 shows the $X$ (horizontal) and $Z$ (vertical) directions of the strata and tunnel lining contour chart. From this figure, the following conclusions can be drawn:

(1) Under the action of self-weight stress, the excavation of the metro tunnel accelerates stratum consolidation, causing both horizontal and vertical displacements. The displacement of each stratum layer is different. Miscellaneous fill develops maximum displacement, and rock strata develop minimum displacement. The maximum horizontal and vertical displacements of the miscellaneous fill are $3.363 \mathrm{~mm}$ and $65.952 \mathrm{~mm}$, respectively. Silty clay has maximum horizontal and vertical displacements of $3.0 \mathrm{~mm}$ and $50.0 \mathrm{~mm}$, respectively. The horizontal and vertical displacements of the strata below the medium weathered rock are less than $1.0 \mathrm{~mm}$ and $10 \mathrm{~mm}$, respectively.

(2) Because of the self-weight stress of the upper layer and the lining itself, the horizontal displacement of the lining's left side is greater than in the right side. The maximum horizontal displacement is $0.925 \mathrm{~mm}$, and the horizontal displacement in the top and bottom of the lining is low. The vertical displacement of the lining top is $5 \mathrm{~mm}$. Because of the highly weathered rock at the bottom, the displacement of the lining bottom is in the range of $2.56-2.75 \mathrm{~mm}$. 
TABLE 2: Vibration velocities and maximum charges of signal shots for different conditions.

\begin{tabular}{lccc}
\hline Condition & Vibration velocity of tunnel $(\mathrm{cm} / \mathrm{s})$ & Vibration velocity of pit edge $(\mathrm{cm} / \mathrm{s})$ & Maximum charge of signal shot $(\mathrm{kg})$ \\
\hline 1st condition & 5 & 105.154 & 3.814 \\
2nd condition & 10 & 210.309 & 12.961 \\
3rd condition & 15 & 315.463 & 26.508 \\
4th condition & 20 & 420.617 & 44.041 \\
\hline
\end{tabular}

TABLE 3: Calculation parameters for rock, soil, and lining.

\begin{tabular}{|c|c|c|c|c|c|c|c|c|}
\hline Name of stratum & Depth (m) & Density $\left(\mathrm{kg} / \mathrm{m}^{3}\right)$ & Poisson's ratio & $C(\mathrm{kPa})$ & $\Phi\left({ }^{\circ}\right)$ & $\begin{array}{l}\text { Bulk modulus } \\
(\mathrm{MPa})\end{array}$ & $\begin{array}{c}\text { Shear modulus } \\
(\mathrm{MPa})\end{array}$ & $\begin{array}{c}\text { Tensile strength } \\
(\mathrm{kPa})\end{array}$ \\
\hline Miscellaneous fill & 4 & 1800 & 0.35 & 10 & 10 & 5.0 & 1.7 & \\
\hline Silty clay & 8 & 1900 & 0.34 & 25 & 15 & 16.7 & 6 & 25 \\
\hline Highly weathered rock & 6 & 2200 & 0.3 & 120 & 25 & 416.7 & 192.3 & 500 \\
\hline Medium weathered rock & 9 & 2400 & 0.27 & 500 & 28 & 1449.3 & 787.4 & 1600 \\
\hline Lining & 0.3 & 2600 & 0.17 & 3200 & 62 & 17424.2 & 14743.6 & 1800 \\
\hline
\end{tabular}

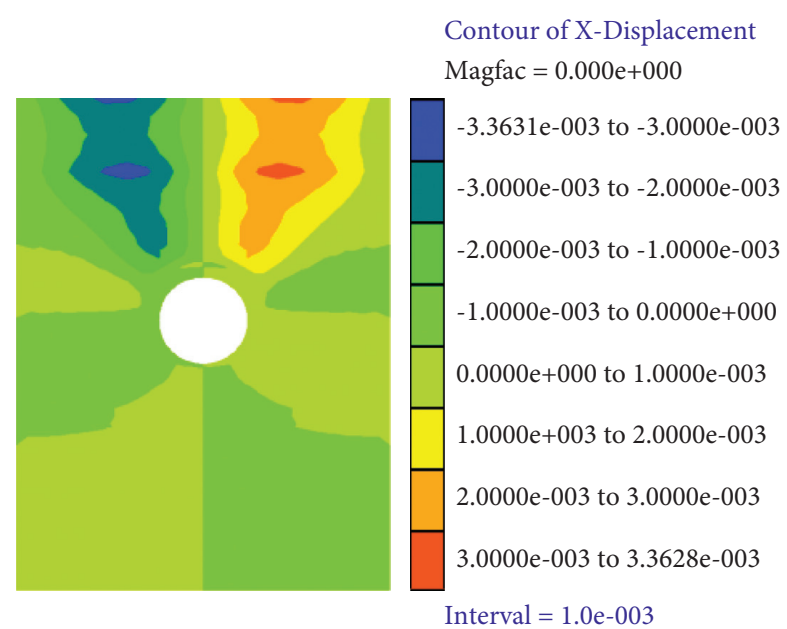

(a)

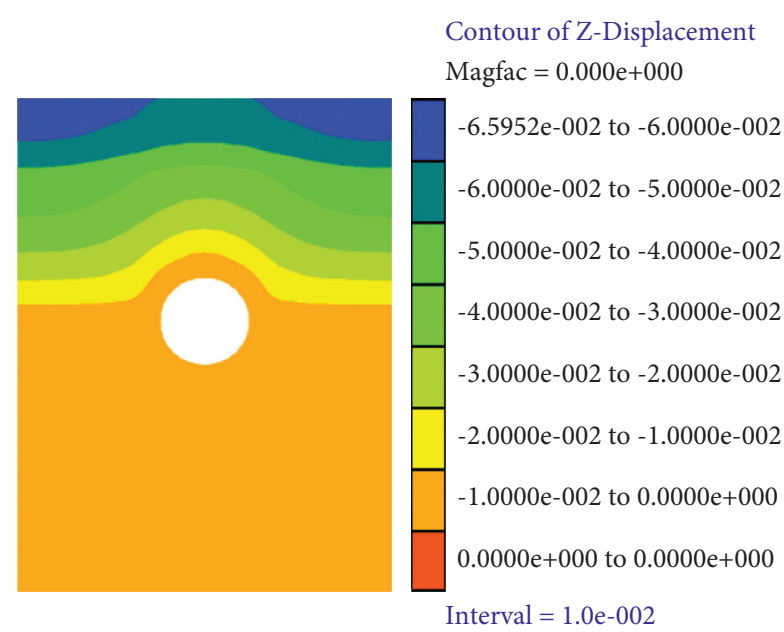

(c)

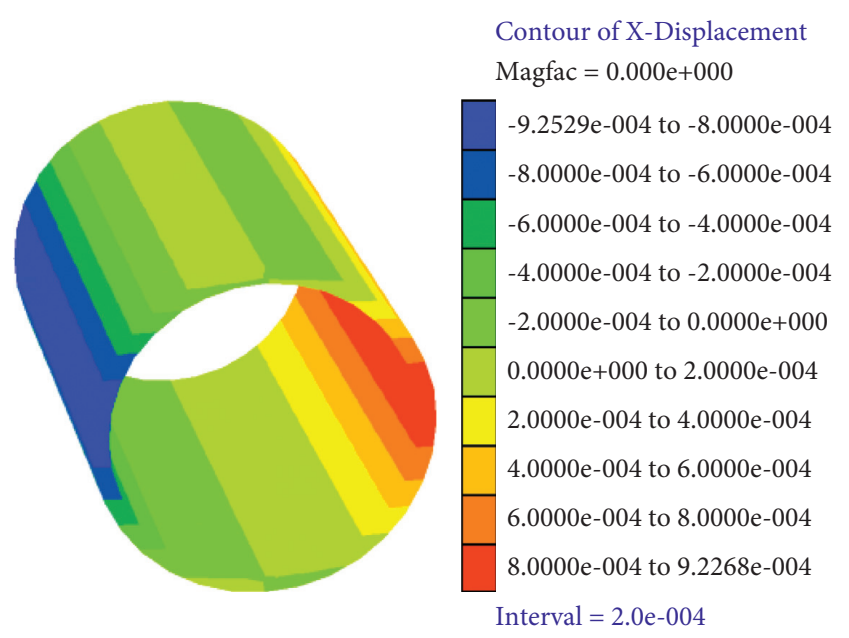

(b)

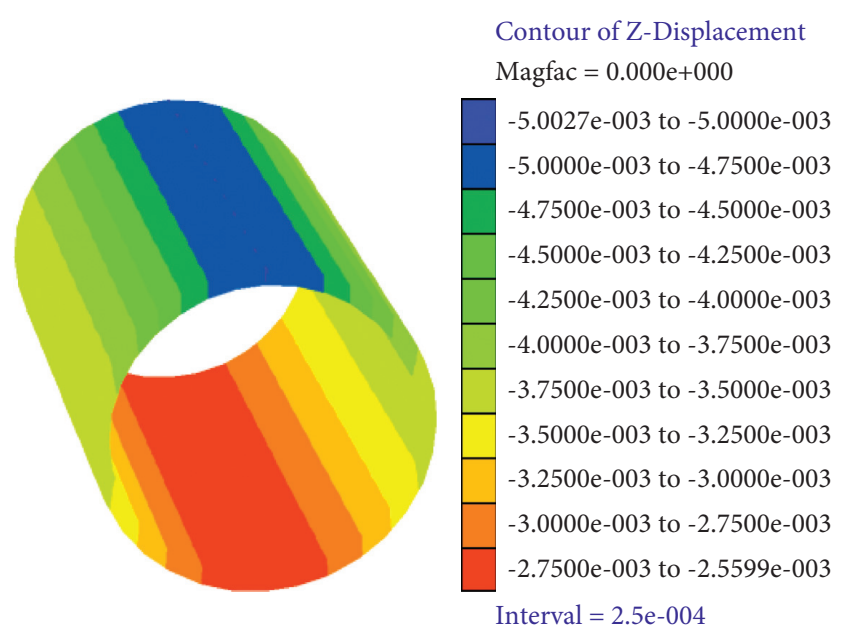

(d)

Figure 7: Displacement contour chart of static load. (a) X-displacement of rock mass. (b) $X$-displacement of lining. (c) Z-displacement of rock mass. (d) Z-displacement of lining. 


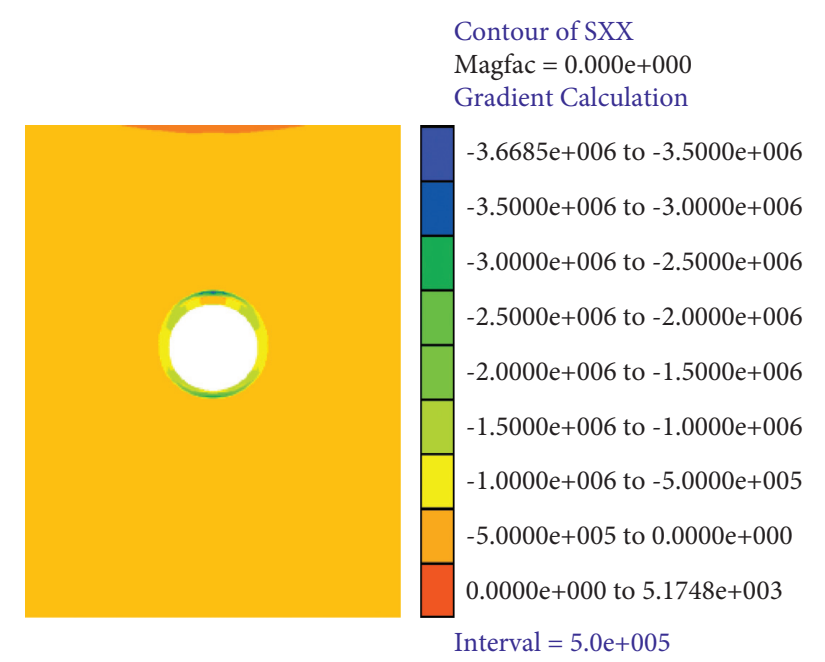

(a)

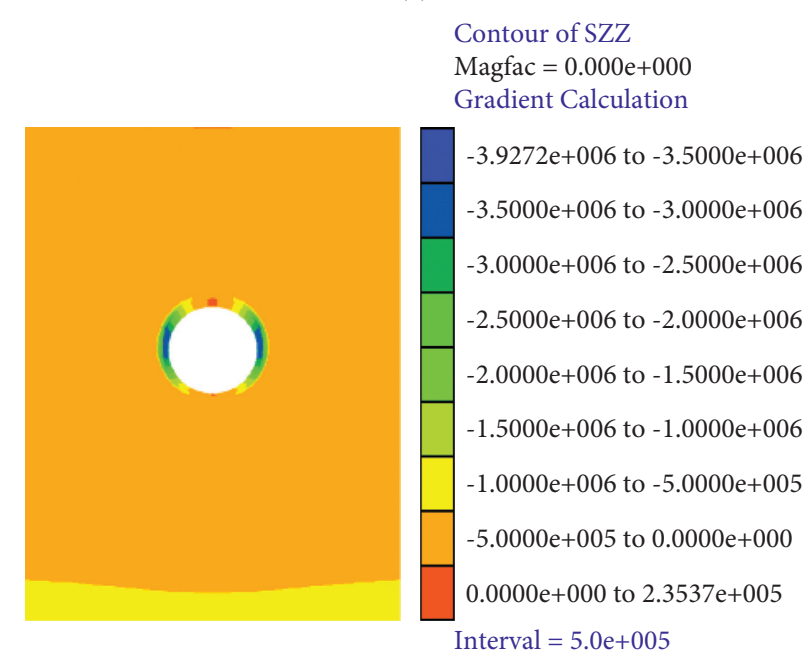

(c)

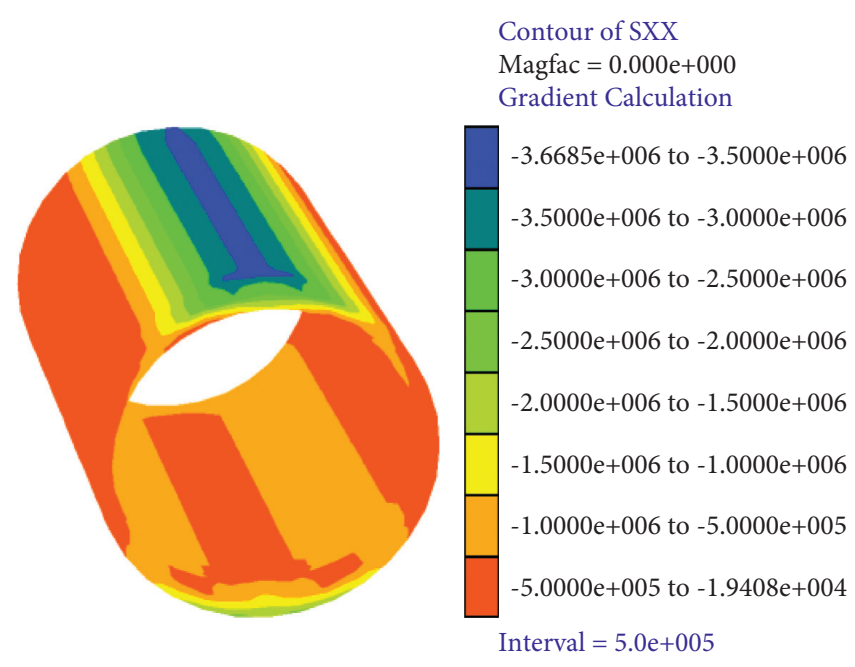

(b)

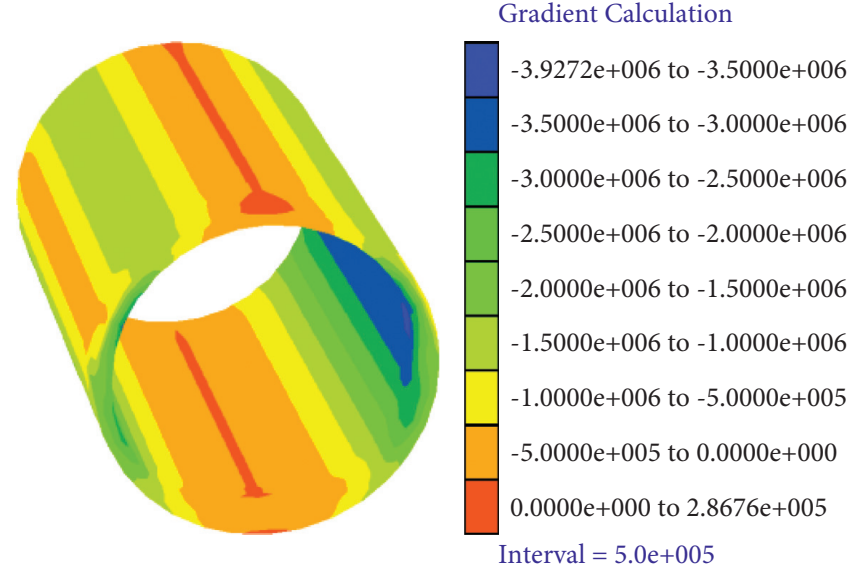

(d)

Figure 8: Stress contour chart of static load. (a) $X$-direction stress of rock mass. (b) $X$-direction stress of lining. (c) $Z$-direction stress of rock mass. (d) Z-direction stress of lining.

This deformation can meet the requirements of the Code for Design of Metro for China [23].

Figure 8 is the stress distribution contour chart of the $X$ (horizontal) and $Z$ (vertical) directions of the strata and the tunnel lining. The contour shows the strata and the tunnel lining, the compression force, and the distributed symmetry with the central vertical line of the lining. The maximum stress of the strata in the $X$ and $Z$ directions is $500 \mathrm{kPa}$, and the maximum horizontal stress at the top of the miscellaneous fill is only $5.175 \mathrm{kPa}$.

In Figure 8(b) and 8(d), the horizontal direction stress of the lining's outside wall is greater than that of the inner wall, and the maximum stress at the lining top and bottom sides is 3.669 MPa. The vertical direction stress of the lining's inner wall is greater than that of the outside wall, with a maximum value of $3.927 \mathrm{MPa}$. Both stresses are far less than the compressive strength of concrete.
It can be seen that the stress and deformation of the stratum and tunnel lining are in the safe range under the action of the self-weight stress.

\subsection{Numerical Simulation Results and Analysis of Dynamic} Load. The geotechnical engineering dynamic analysis using FLAC3D is based on the static analysis results. Before the dynamic calculation, the displacement of each direction is set to zero. Then, the boundary conditions are set, the blasting dynamic load is applied, and the calculation is carried out. The stress and displacement of the rock mass and lining under different blasting dynamic loads are calculated. There are too many images of calculation results for four kinds of conditions, and the distribution law is almost quite similar. Therefore, only the calculations of the results for the first and fourth conditions are listed, as shown in Figures 9 and 10. 


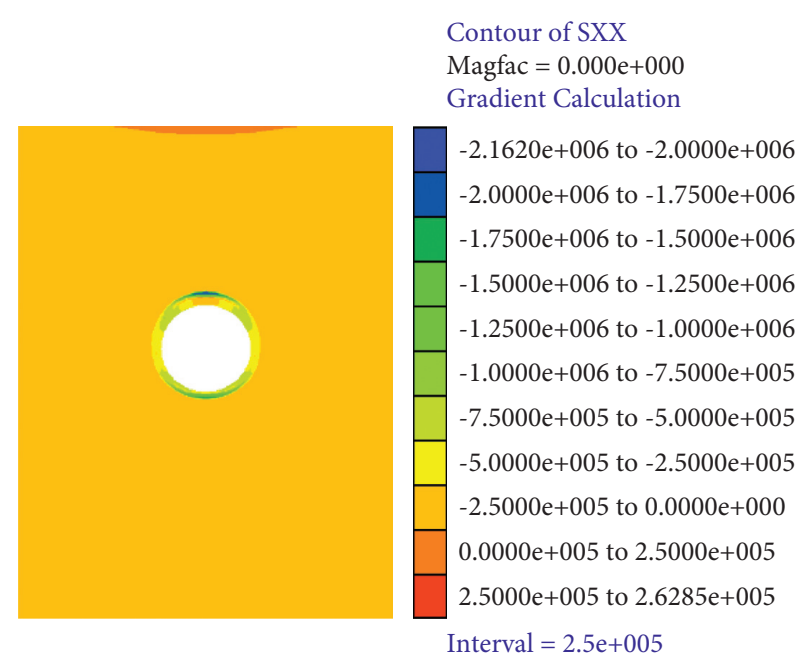

(a)

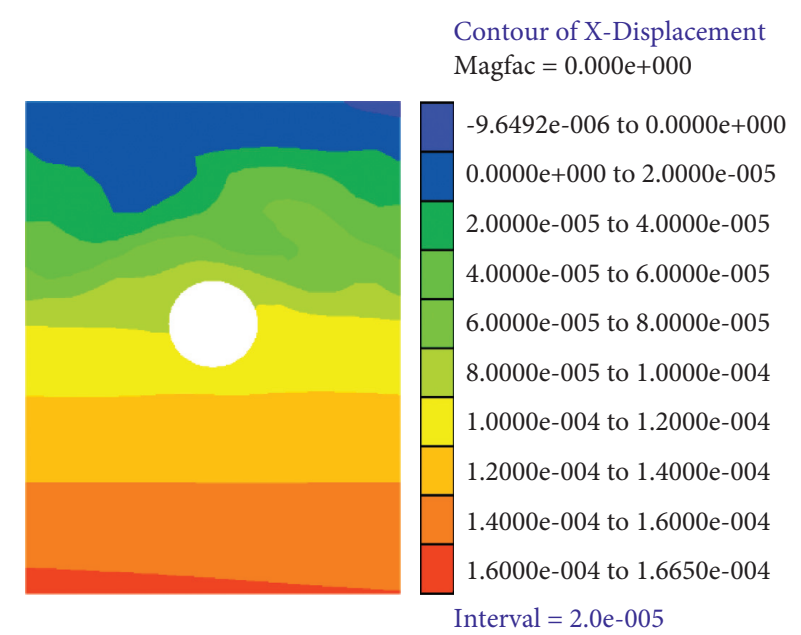

(c)

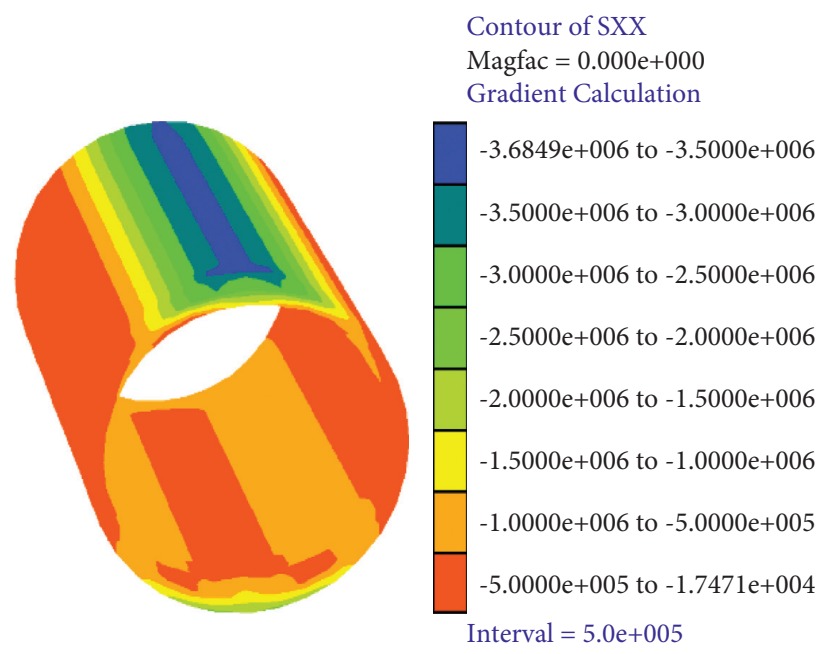

(e)

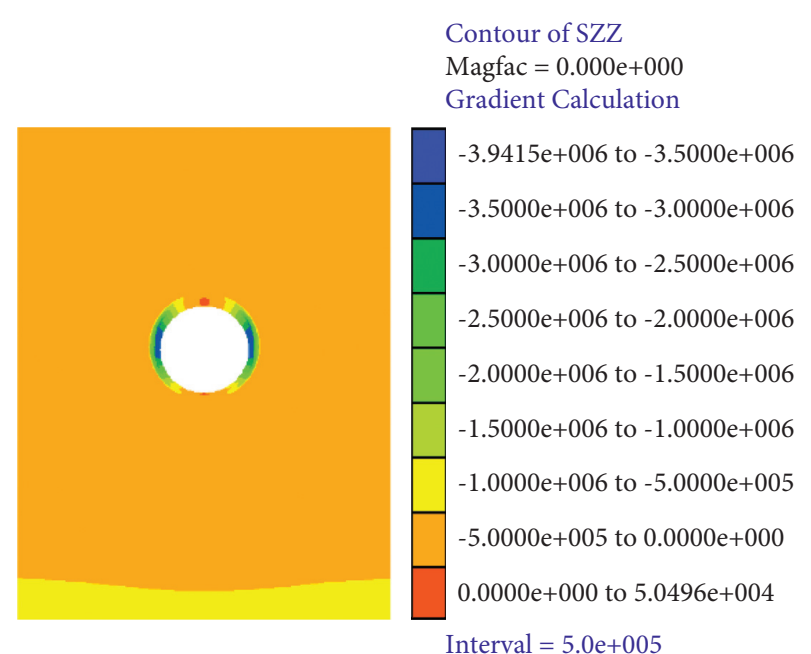

(b)

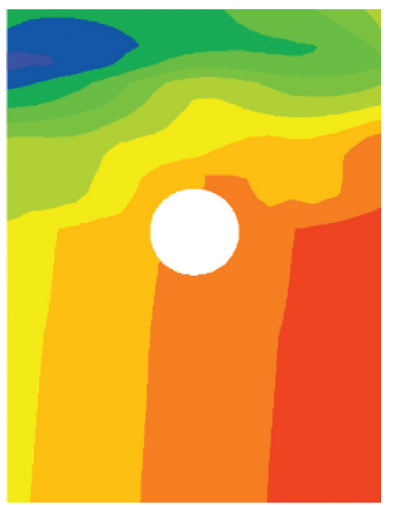

Contour of Z-Displacement Magfac $=0.000 \mathrm{e}+000$

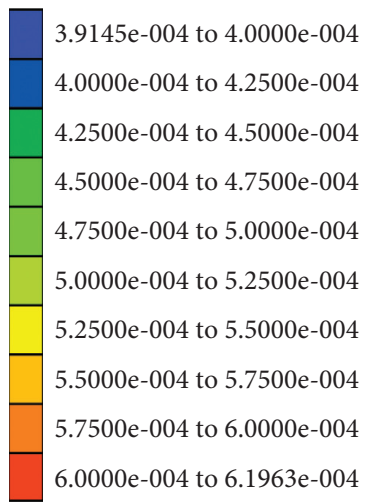

Interval $=2.5 \mathrm{e}-005$

(d)

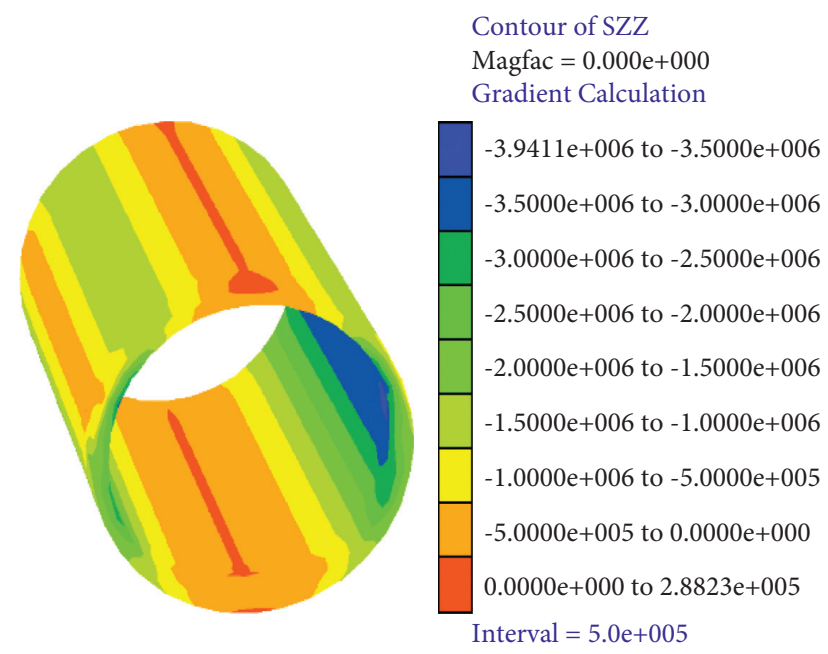

(f)

Figure 9: Continued. 


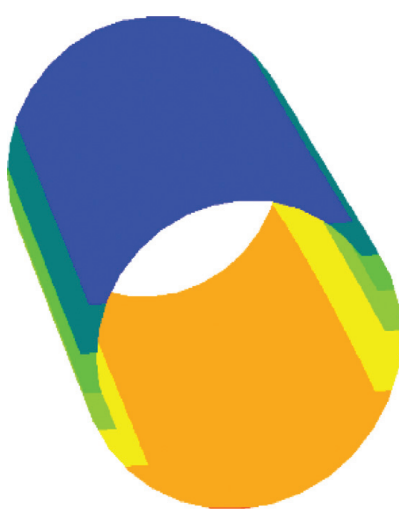

Contour of X-Displacement Magfac $=0.000 \mathrm{e}+000$

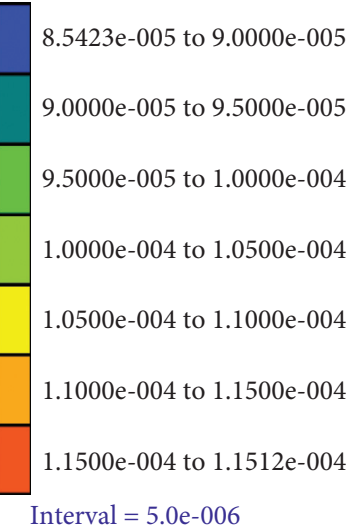

(g)

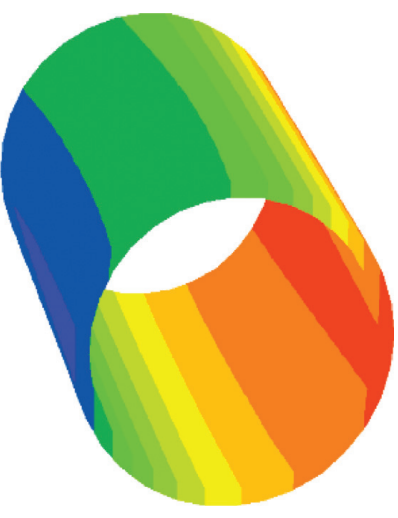

Contour of Z-Displacement Magfac $=0.000 \mathrm{e}+000$

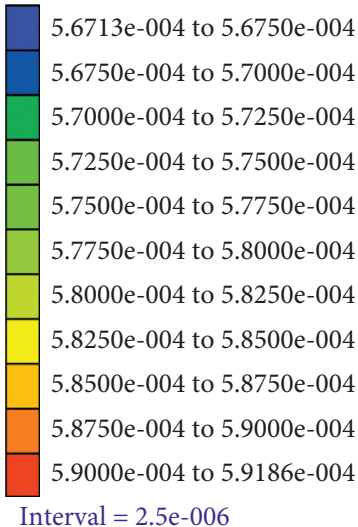

(h)

FIGURE 9: Displacement and stress contour chart of the 1st condition. (a) $X$-direction stress of the model. (b) $Z$-direction stress of the model. (c) $X$-displacement of the model. (d) Z-displacement of the model. (e) $X$-direction stress of the lining. (f) $Z$-direction stress of the lining. (g) $X$-displacement of the lining. (h) $Z$-displacement of the lining.

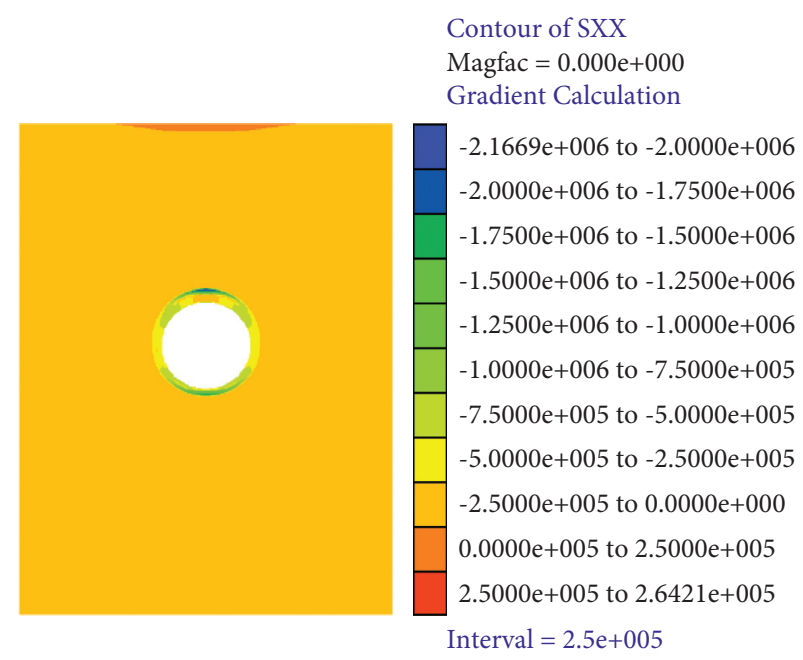

(a)

Contour of X-Displacement Magfac $=0.000 \mathrm{e}+000$

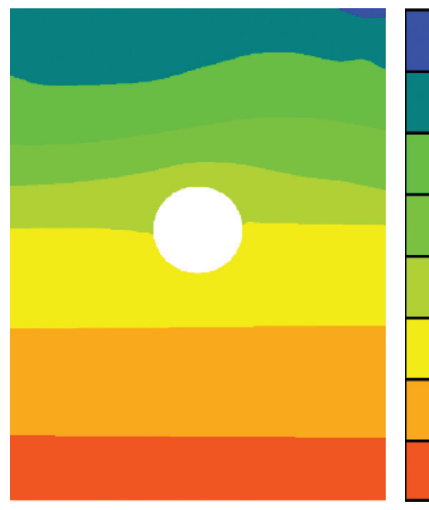

$-2.9630 \mathrm{e}-005$ to $0.0000 \mathrm{e}+000$ $0.0000 \mathrm{e}+000$ to $1.0000 \mathrm{e}-004$ $1.0000 \mathrm{e}-004$ to $2.0000 \mathrm{e}-004$ $2.0000 \mathrm{e}-004$ to $3.0000 \mathrm{e}-004$ $3.0000 \mathrm{e}-004$ to $4.0000 \mathrm{e}-004$ $4.0000 \mathrm{e}-004$ to $5.0000 \mathrm{e}-004$ $5.0000 \mathrm{e}-004$ to $6.0000 \mathrm{e}-004$ $6.0000 \mathrm{e}-004$ to $6.6069 \mathrm{e}-004$ Interval $=1.0 \mathrm{e}-004$

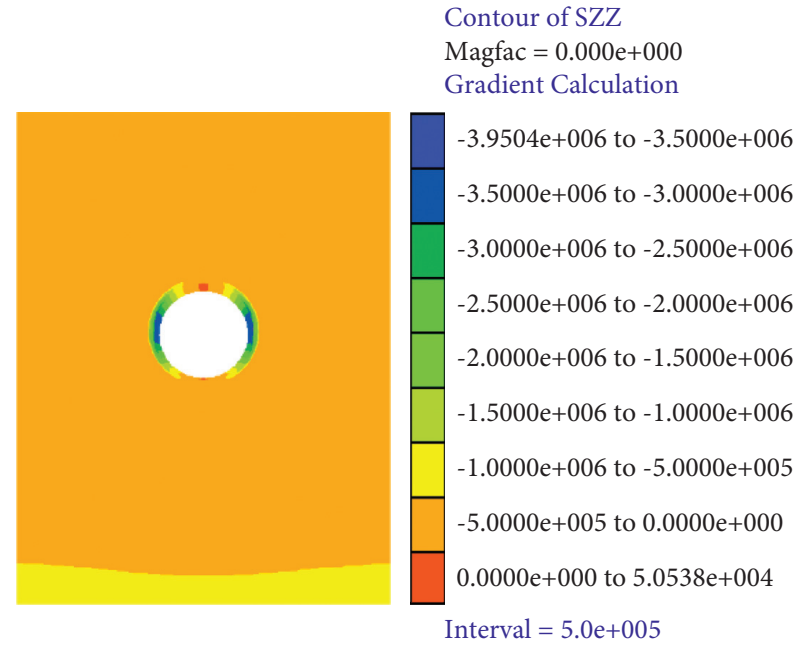

(b)

Contour of Z-Displacement Magfac $=0.000 \mathrm{e}+000$
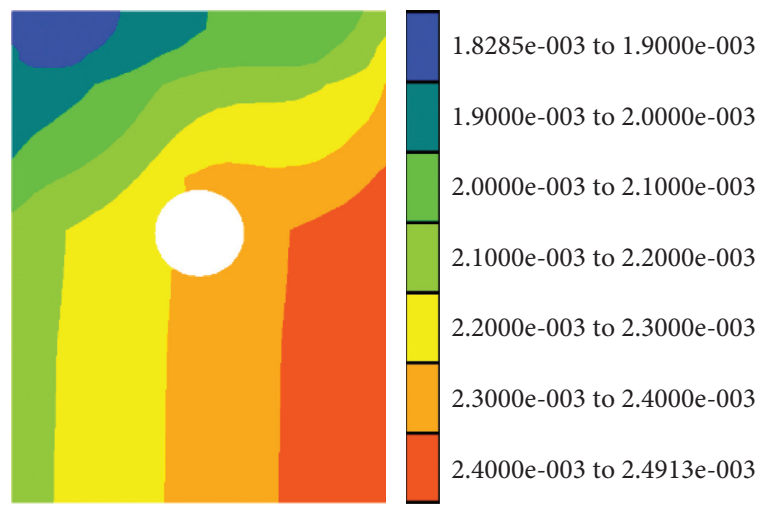

Interval $=1.0 \mathrm{e}-004$

(c)

Figure 10: Continued. 


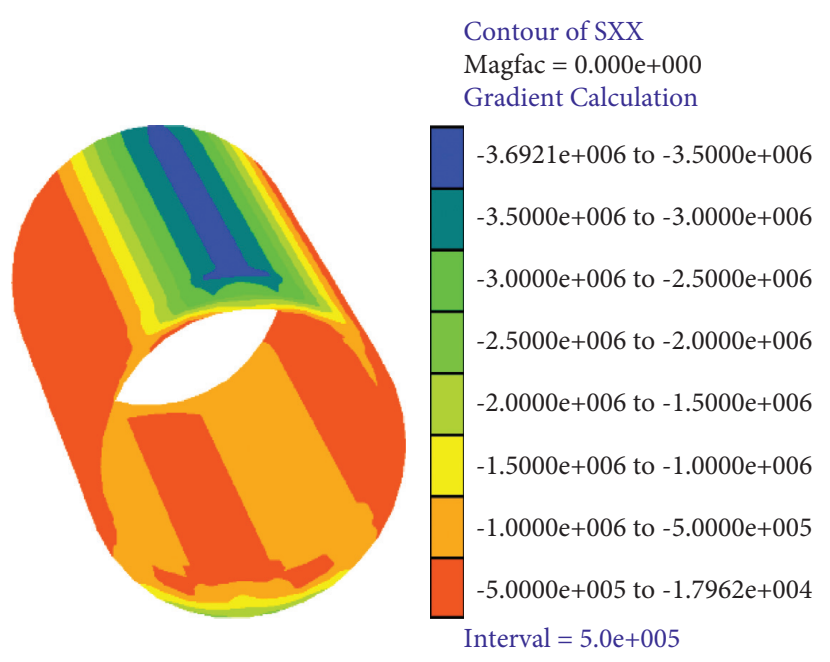

(e)

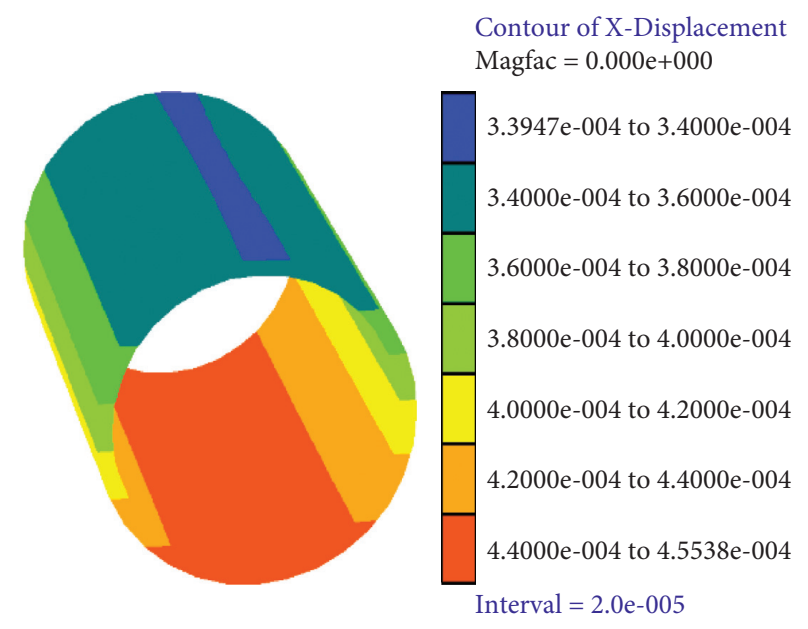

(g)

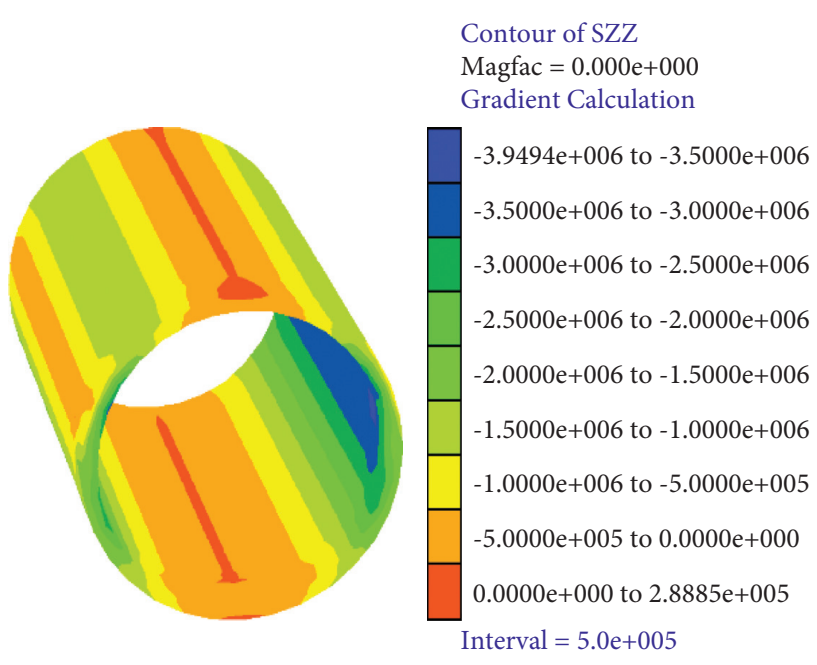

(f)

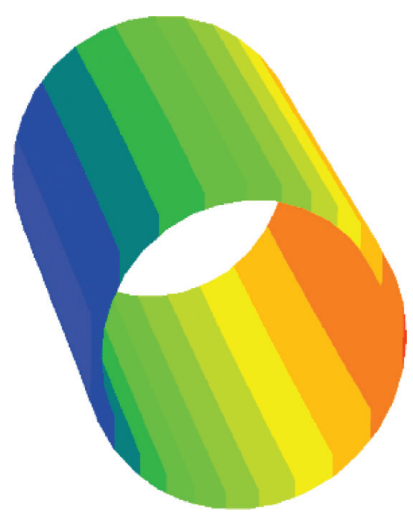

Contour of Z-Displacement Magfac $=0.000 \mathrm{e}+000$

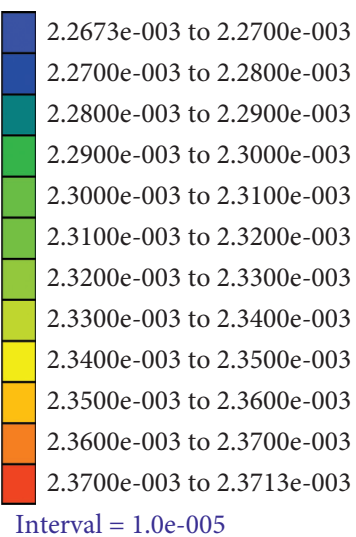

(h)

Figure 10: Stress contour chart of the fourth condition. (a) $X$-direction stress of the model. (b) $Z$-direction stress of the model. (c) $X$ displacement of the model. (d) $Z$-displacement of the model. (e) $X$-direction stress of the lining. (f) $Z$-direction stress of the lining. (g) $X$ displacement of the lining. (h) Z-displacement of the lining.

Figure 9 shows that after applying the blasting dynamic load, the deformation of the metro tunnel lining in the geotechnical engineering model is quite small, with a magnitude of $10^{-4} \mathrm{~m}$, so the deformation will not have an adverse effect. For the rock mass, because of the dynamic load in the lower-left part of the model, a relatively large displacement appears in the region, with maximum horizontal and vertical displacements of $1.666 \times 10^{-4} \mathrm{~m}$ and $6.196 \times 10^{-4} \mathrm{~m}$, respectively.

For the metro tunnel lining, because of the blasting dynamic load applied in the bottom left of the model, the deformations around the inside and outside of the lining are not the same.

(1) The horizontal displacement is greater at the top, and the displacement at the bottom of the outer wall is greater than that of the wall. The maximum horizontal displacement is $1.153 \times 10^{-4} \mathrm{~m}$, and at the bottom of the inside, the displacement is $1.151 \times 10^{-4} \mathrm{~m}$.
(2) For the vertical displacement, the right side is larger than the left. For the right side wall, the maximum displacement of outer is $5.924 \times 10^{-4} \mathrm{~m}$ and the maximum displacement of inner is $5.912 \times 10^{-4} \mathrm{~m}$, the maximal displacement of the left side is $5.675 \times 10^{-4} \mathrm{~m}$.

As shown in Figure 10, after applying the blasting dynamic load, the metro tunnel lining, rock, and soil mass are mainly compressive stress. The stresses around the interior and exterior walls are different. The maximum horizontal stress is $3.685 \mathrm{MPa}$ on the top of the lining walls and $2.575 \mathrm{MPa}$ for the lining at the bottom of the outer wall. The maximum vertical stress in the lining of the left side is $3.941 \mathrm{MPa}$, and the compressive stress for the lining of the right side is $3.941 \mathrm{MPa}$.

4.3. Analysis of Numerical Simulation Results in Different Conditions. To analyze the relationship between the applied 
blasting dynamic load and the maximum displacement, we summarize the stress of the tunnel lining, the maximum displacement, and the stress of the lining under static conditions and a dynamic load in Table 4, from which we can see the following:

(1) The stress and displacement of the metro tunnel lining increase as long as the blasting dynamic load strength improves.

(2) Compared to the static load, after applying the blasting dynamic load, the displacements of the subway tunnel lining are positive. This is shown in the tunnel lining toward the right and upward from the blasting dynamic load. The displacement of the 4th condition is the largest, with a vertical displacement of $23.719 \times 10^{-4} \mathrm{~m}$ and a horizontal displacement of $4.554 \times 10^{-4} \mathrm{~m}$, both within the allowable range [13].

(3) After applying the blasting dynamic load, the maximum stress of the lining increases slightly. The maximum stress growth rates for the different conditions are shown in Table 5, from which we see that the growth rate of the horizontal stress on the top is greatly influenced by the blasting dynamic load compared to the rate of the other stress and the growth rate of the horizontal stress in the bottom is less affected by the blasting dynamic load.

The relationship between the tunnel lining maximum displacement and the vibration velocity for different conditions is shown in Figure 11, from which we can see that the vertical displacement is more vulnerable than the horizontal displacement to the impact of the blasting dynamic load.

According to Figure 11, the displacement of the tunnel lining increases linearly with the vibration velocity.

The vertical displacement of the lining right side is

$\delta_{z-r}=1.1884 v-0.0251$ (correlation coefficient is 0.99$)$,

the vertical displacement of the lining left side is

$\delta_{z-1}=1.1365 v($ correlation coefficient is 0.99$)$,

the horizontal displacement of the lining bottom is

$$
\delta_{x-b}=0.2271 v-0.0131 \text { (correlation coefficient is } 0.99 \text { ), }
$$

and the horizontal displacement of the lining top is

$$
\left.\delta_{x-t}=0.1677 v-0.0526 \text { (correlation coefficient is } 0.99\right) \text {, }
$$

where $\delta_{z-r}$ is the vertical displacement in the right side of the lining, $10^{-4} \mathrm{~m} ; \delta_{z-1}$ is the vertical displacement in the left side of the lining, $10^{-4} \mathrm{~m} ; \delta_{x-b}$ is the horizontal displacement in the bottom of the lining, $10^{-4} \mathrm{~m} ; \delta_{x-t}$ is the horizontal displacement in the top of lining, $10^{-4} \mathrm{~m}$; and $v$ is the blasting vibration velocity of the lining, $\mathrm{cm} / \mathrm{s}$.
From Figure 11 and equations (5)-(8), it can be found that the vertical displacement of the right side of the metro tunnel lining increases when the blasting vibration velocity is fastest, so the vertical displacement of the lining top can be used as a control index of the influence of the blasting construction on the metro tunnel. As calculated by equation (5), the corresponding vibration velocity of the lining displacement of $1.0 \mathrm{~mm}$ is $8.436 \mathrm{~cm} / \mathrm{s}$, and the maximum initiation explosive charge is $5.556 \mathrm{~kg}$. It is more manageable than equation (1) according to the allowable limit vibration speeds; in equation (1), the charge can only be calculated according to the allowable vibration velocity limit of $2.0 \mathrm{~cm} / \mathrm{s}$ or $15.0 \mathrm{~cm} / \mathrm{s}$.

\section{Optimization of Blasting Parameters}

During blasting construction, the maximum initiation explosive charge for the design of the blasting parameters is $5.0 \mathrm{~kg}$.

\subsection{Blasting Parameters for the Manual Digging Pile}

5.1.1. Blast Holes Layout. The diameters of the manual digging piles range from 1.50 to $2.80 \mathrm{~m}$, and their thickness is $0.3 \mathrm{~m}$. The diameter of the blasting space ranges from $1.80 \mathrm{~m}$ to $3.10 \mathrm{~m}$.

Depth of hole: $L=1.0 \mathrm{~m}$.

Depth of cut hole: $L_{1}=(1.2 \sim 1.4) L=1.2-1.4 \mathrm{~m}$. The chosen value is $1.2 \mathrm{~m}$.

5.1.2. Calculation of Explosive Charge. The explosive charge is calculated as follows:

$$
Q_{1}=q_{1} S L \eta
$$

where $Q_{1}$ is the charge of a single blast in the pile in $\mathrm{kg}, q_{1}$ is the explosive consumption of the unit volume rock in $\mathrm{kg} / \mathrm{m}^{3}$, $S$ is the blast area in $\mathrm{m}^{2}, L$ is the depth of the blast hole in $m$, and $\eta$ is the blast hole utilization ratio.

The explosive diameter is $32 \mathrm{~mm}$ in the field. Taking the $1.50 \mathrm{~m}$ diameter of the manual digging pile as an example (the excavation diameter is $1.8 \mathrm{~m}$ ), the explosive consumption of rock is $2.0 \mathrm{~kg} / \mathrm{m}^{3}$, so the total charge of a single blast is calculated as follows:

$$
Q_{1}=2.0 \times 2.54 \times 1.2 \times 0.85=4.32 \mathrm{~kg} .
$$

In actual construction, blasting parameters are shown in Table 6, and Figure 12 shows holes' arrangement.

5.1.3. Charge Structure and Initiation Style. Boring priming is adopted. A reasonable initiation time can improve the blasting effect and reduce blasting vibration. The initiation sequence is as follows: the delay time is $25 \mathrm{~ms}$ for cut holes, 75 and $110 \mathrm{~ms}$ for cut spreader holes, and $200-460 \mathrm{~ms}$ for contour holes. 
TABLE 4: Maximum displacement and stress of linings for different conditions.

\begin{tabular}{lcccccrrrr}
\hline Condition & Velocity $(\mathrm{cm} / \mathrm{s})$ & \multicolumn{2}{c}{$\begin{array}{c}\text { Vertical } \\
\text { displacement } \\
\left(10^{-4} \mathrm{~m}\right)\end{array}$} & \multicolumn{2}{c}{$\begin{array}{c}\text { Horizontal } \\
\text { displacement } \\
\left(10^{-4} \mathrm{~m}\right)\end{array}$} & \multicolumn{2}{c}{$\begin{array}{c}\text { Z-direction stress } \\
(\mathrm{MPa})\end{array}$} & \multicolumn{2}{c}{$\begin{array}{c}X \text {-direction stress } \\
(\mathrm{MPa})\end{array}$} \\
& & Top & Bottom & Left & Right & Top & Bottom & Left & Right \\
\hline Static load & 0 & -50.03 & -27.5 & -9.253 & 9.239 & -3.927 & -3.927 & -3.669 & -2.568 \\
1st condition & 5 & 5.675 & 5.924 & 0.9 & 1.153 & -3.942 & -3.941 & -3.685 & -2.575 \\
2nd condition & 10 & 11.35 & 11.821 & 1.70 & 2.275 & -3.945 & -3.944 & -3.686 & -2.577 \\
3rd condition & 15 & 17.1 & 17.855 & 2.60 & 3.429 & -3.950 & -3.949 & -3.692 & -2.579 \\
4th condition & 20 & 22.7 & 23.719 & 3.395 & 4.554 & -3.950 & -3.949 & -3.692 & -2.579 \\
\hline
\end{tabular}

TABLE 5: Stress growth rates of linings for different conditions (unit: \%).

\begin{tabular}{lcccc}
\hline Condition & \multicolumn{2}{c}{ Z-direction stress } & \multicolumn{2}{c}{$X$-direction stress } \\
\hline 1st condition & Left & Right & Top & 0.447 \\
2nd condition & 0.369 & 0.354 & 0.477 & 0.277 \\
3rd condition & 0.458 & 0.438 & 0.632 & 0.374 \\
4th condition & 0.593 & 0.568 & 0.643 & 0.460 \\
\hline
\end{tabular}

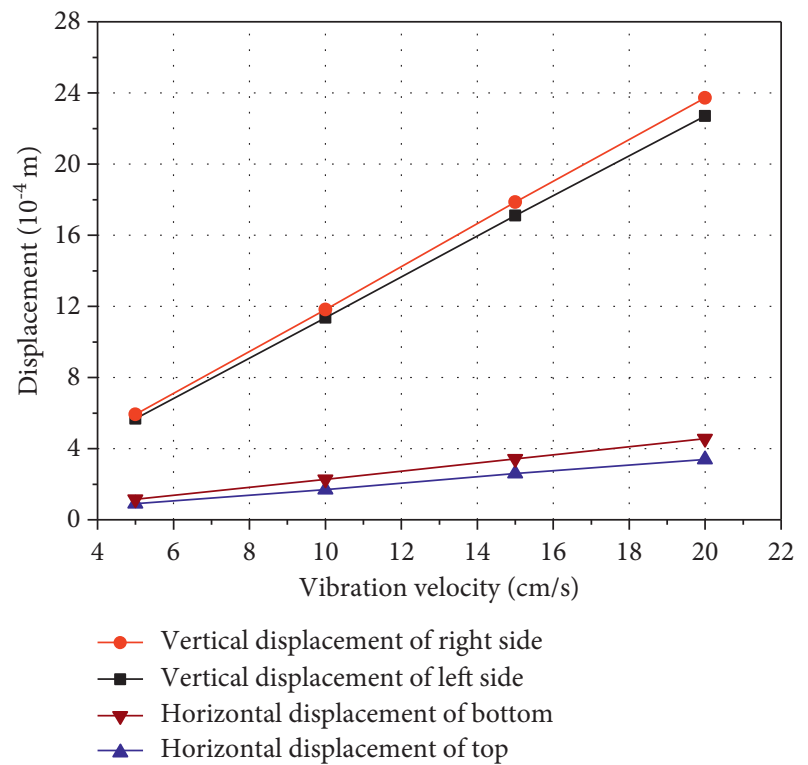

Figure 11: Relationship between maximum displacement and vibration velocity of the lining.

TABLE 6: Blasting parameters of a pile with net diameters of $1.5 \mathrm{~m}$.

\begin{tabular}{|c|c|c|c|c|c|c|c|}
\hline No. of circle & No. of hole & Diameter of circle $(\mathrm{mm})$ & $\begin{array}{l}\text { Depth of hole } \\
(\mathrm{m})\end{array}$ & $\begin{array}{l}\text { Distance of hole } \\
(\mathrm{mm})\end{array}$ & $\begin{array}{c}\text { Distance of circle } \\
(\mathrm{mm})\end{array}$ & $\begin{array}{l}\text { Quantity of hole } \\
\text { (unit) }\end{array}$ & $\begin{array}{l}\text { Charge of unit } \\
\text { hole }(\mathrm{kg})\end{array}$ \\
\hline I & $1-4$ & 800 & 1.20 & 560 & & 4 & 0.50 \\
\hline II & $5-12$ & 1600 & 1.0 & 530 & 300 & 8 & 0.30 \\
\hline Total & & & & & & 12 & 4.40 \\
\hline
\end{tabular}

\subsection{Blasting Parameters for Foundation Pit Rock Mass}

5.2.1. Blast Hole Layout. Short-hole bench blasting of 2.0-3.0 $\mathrm{m}$ in height is adopted for foundation pit excavation, and the holes of nearby rows form a triangle.
5.2.2. Calculation of Explosive Charge. The explosive charge is calculated as follows:

$$
Q_{2}=q_{2} a b H,
$$




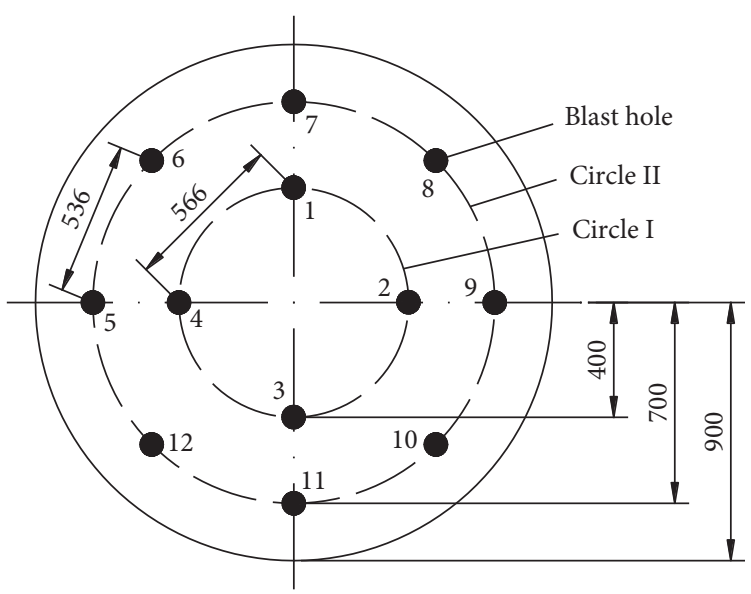

FIgURE 12: Blast-hole layout of a pile with net diameters of $1.5 \mathrm{~m}$ (unit: $\mathrm{mm}$ ).

TABLE 7: Blasting parameters of the foundation pit.

\begin{tabular}{lcccc}
\hline Depth of hole $(\mathrm{m})$ & $a \times b(\mathrm{~m})$ & $q_{2}\left(\mathrm{~kg} \cdot \mathrm{m}^{-3}\right)$ & Charge of unit hole $(\mathrm{kg})$ & Length of stemming $(\mathrm{m})$ \\
\hline 2.0 & $1.0 \times 0.9$ & 0.45 & 0.8 & 1.2 \\
2.5 & $1.1 \times 0.9$ & 0.45 & 1.1 & 1.4 \\
3.0 & $1.2 \times 1.0$ & 0.45 & 1.6 & 1.4 \\
\hline
\end{tabular}

where $Q_{2}$ is the charge of a single blast in the foundation pit in $\mathrm{kg}, q_{2}$ is the explosive consumption of the unit volume rock in $\mathrm{kg} / \mathrm{m}^{3}, a$ is the distance between the blast holes in $m$, $b$ is the distance between nearby rows in $m$, and $H$ is the depth of the blast hole in $m$.

Blasting parameters of different depths can be calculated using equation (11). These are shown in Table 7.

The parameters are adjusted during construction according to the properties of the rock, blasting effect, and blasting vibration.

\section{Conclusions}

In this paper, a metro tunnel lining adjacent to the excavation blasting of a foundation pit is taken as a case study. Through the combination of vibration monitoring and numerical calculation, the effects of foundation pit blasting on metro tunnel lining are studied and analyzed. The main conclusions are as follows:

(1) Under the blasting dynamic load, the tunnel lining displacement is within the allowable range, and the displacement increases linearly with the vibration velocity of the dynamic load. The vertical displacement is greater than the horizontal displacement and is influenced more by the blasting dynamic load.

(2) Compared to the static load condition, the stress amplitude of the dynamic load condition increases by $0.227 \%-0.643 \%$. The horizontal stress at the top of the tunnel lining is greatly influenced by the blasting dynamic load, and the horizontal stress at the bottom is less affected by the blasting dynamic load.

(3) The tunnel lining vertical displacement is proposed as the control index of the influence of blasting construction on the metro tunnel.
(4) The prediction formula of metro lining PPV is established; according to the formula, blast hole layout and explosive charge of unit hole for the manual digging pile and foundation pit rock mass are optimized.

\section{Data Availability}

The data used to support the findings of this study are available from the corresponding author upon request.

\section{Conflicts of Interest}

The authors declare that there are no known conflicts of interest associated with this publication.

\section{Acknowledgments}

This work was supported by the National Natural Science Foundation of China (no. 51404010), the Natural Science Foundation of Anhui Province (no. 2008085ME163), and the Postdoctoral Science Foundation of Anhui Province (no. 2018B282).

\section{References}

[1] S.-S. Lin, S.-L. Shen, A. Zhou, and Y.-S. Xu, "Risk assessment and management of excavation system based on fuzzy set theory and machine learning methods," Automation in Construction, vol. 122, Article ID 103490, 2021.

[2] S.-L. Shen, Y.-X. Wu, and A. Misra, "Calculation of head difference at two sides of a cut-off barrier during excavation dewatering," Computers and Geotechnics, vol. 91, pp. 192-202, 2017.

[3] K. Elbaz, S.-L. Shen, Y. Tan, and W.-C. Cheng, "Investigation into performance of deep excavation in sand covered karst: a 
case report," Soils and Foundations, vol. 58, no. 4, pp. 1042-1058, 2018.

[4] Y.-F. Jin, Z.-Y. Yin, W.-H. Zhou, and X. Liu, "Intelligent model selection with updating parameters during staged excavation using optimization method," Acta Geotechnica, vol. 15, no. 9, pp. 2473-2491, 2020.

[5] Q.-L. Cui, S.-L. Shen, Y.-S. Xu, H.-N. Wu, and Z.-Y. Yin, "Mitigation of geohazards during deep excavations in karst regions with caverns: a case study," Engineering Geology, vol. 195, pp. 16-27, 2015.

[6] X. Xia, H. Li, Y. Liu, and C. Yu, "A case study on the cavity effect of a water tunnel on the ground vibrations induced by excavating blasts," Tunnelling and Underground Space Technology, vol. 71, pp. 292-297, 2018.

[7] S. Koneshwaran, D. P. Thambiratnam, and C. Gallage, "Blast response of segmented bored tunnel using coupled SPH-FE method," Structure, vol. 2, no. 2, pp. 58-71, 2015.

[8] E. M. El Koursi, J.-L. Bruyelle, R. Seddon, and C. O’Neill, "Design solutions to improve resilience of metro vehicle to blast events," Transportation Research Part A: Policy and Practice, vol. 118, pp. 280-291, 2018.

[9] J.-H. Shin, H.-G. Moon, and S.-E. Chae, "Effect of blast-induced vibration on existing tunnels in soft rocks," Tunnelling and Underground Space Technology, vol. 26, no. 1, pp. 51-61, 2011.

[10] U. Ozer, "Environmental impacts of ground vibration induced by blasting at different rock units on the KadikoyKartal metro tunnel," Engineering Geology, vol. 100, no. 1-2, pp. 82-90, 2008.

[11] I. Ocak and N. Bilgin, "Comparative studies on the performance of a roadheader, impact hammer and drilling and blasting method in the excavation of metro station tunnels in Istanbul," Tunnelling and Underground Space Technology, vol. 25, no. 2, pp. 181-187, 2010.

[12] A. De, A. N. Morgante, and T. F. Zimmie, "Numerical and physical modeling of geofoam barriers as protection against effects of surface blast on underground tunnels," Geotextiles and Geomembranes, vol. 44, no. 1, pp. 1-12, 2016.

[13] D. Park and S. Jeon, "Reduction of blast-induced vibration in the direction of tunneling using an air-deck at the bottom of a blasthole," International Journal of Rock Mechanics and Mining Sciences, vol. 47, no. 5, pp. 752-761, 2010.

[14] R. Masoudi and M. Sharifzadeh, "Reinforcement selection for deep and high-stress tunnels at preliminary design stages using ground demand and support capacity approach," International Journal of Mining Science and Technology, vol. 28, no. 4, pp. 573-582, 2018.

[15] V. R. Feldgun, Y. S. Karinski, and D. Z. Yankelevsky, "The effect of an explosion in a tunnel on a neighboring buried structure," Tunnelling and Underground Space Technology, vol. 44, pp. 42-55, 2014.

[16] S.-S. Lin, S.-L. Shen, A. Zhou, and Y.-S. Xu, "Novel model for risk identification during karst excavation," Reliability Engineering \& System Safety, vol. 209, Article ID 107435, 2021.

[17] Q. Qin and J. Zhang, "Vibration control of blasting excavation of large cross-section highway tunnel over metro line," Arabian Journal of Geosciences, vol. 13, no. 17, Article ID 868, 2020.

[18] N. Jiang, T. Gao, C. Zhou, and X. Luo, "Effect of excavation blasting vibration on adjacent buried gas pipeline in a metro tunnel," Tunnelling and Underground Space Technology, vol. 81, pp. 590-601, 2018.

[19] N. Jiang, B. Zhu, X. He, C. Zhou, X. Luo, and T. Wu, "Safety assessment of buried pressurized gas pipelines subject to blasting vibrations induced by metro foundation pit excavation," Tunnelling and Underground Space Technology, vol. 102, Article ID 103448, 2020.

[20] Z. Zhang, Y. Li, S. Wang, H. Zhang, and Y. Qian, “Assessing and controlling of boulder deep-hole blasting-induced vibrations to minimize impacts to a neighboring metro shaft," Archives of Civil and Mechanical Engineering, vol. 21, no. 2, Article ID 66, 2021.

[21] S. Liu, H. Su, and X. Si, "Optimization study on blasting scheme of metro stations subsurface excavating oblique through pile foundations of existing elevated light rail lines," Advances in Mechanical Engineering, vol. 12, no. 6, Article ID 1687814020933063, 2020.

[22] Standard of Guangzhou, Technical Specification for Retaining and Protection of Building Foundation Excavations in Guangzhou Area (GJB 02-98), (in China), p. 76, Guangzhou Institute of Geochemistry, Guangzhou, ChinaGuangzhou Institute of Geochemistry, 1998.

[23] Standard of Guangzhou, Code for Design of Metro for China, Guangzhou Institute of Geochemistry, Guangzhou, China, in China, 2013. 